\title{
Der blinde Fleck der zweiten Moderne. Globale Finanzmärkte und die Theorie reflexiver Modernisierung*
}

\section{Von Daniel Mügge}

Für die Theorie reflexiver Modernisierung bilden Finanzmärkte einen Sonderfall. In vielen anderen Bereichen sozialen Lebens kann man zwischen zwei idealtypischen Phasen von Modernisierung unterscheiden (Beck/Giddens/Lash 1994; Beck/Bonß/Lau 2001): In der ersten Moderne ist die traditionelle Gesellschaft das Objekt von Modernisierung. Diese Trennung der Modernisierung von ihren Objekten fußt auf einer klaren Unterscheidung zwischen der >Natur $<$ - den Objekten sozialen Handelns - und dem Handeln selbst, das versucht, diese Natur unter modernen Vorzeichen zu prägen und anzupassen. In der zweiten Moderne kollabiert diese Unterscheidung. Die moderne Gesellschaft wird selbst zum Gegenstand von Modernisierung. Das Objekt menschlichen Handels ist nicht länger eine ihm äußerliche Natur, sondern das Produkt menschlichen Handelns selbst.

Allein, für Finanzmärkte gilt die Unterscheidung zwischen erster und zweiter Moderne nicht ohne weiteres. Geld - in welcher Form dann auch - ist schon immer das Produkt sozialen Handels gewesen. Weiter noch, Finanzmärkte werden seit dem Beginn ihrer Entwicklung von Krisen geplagt, also genau der Art von ungeplanten und unerwünschten Nebeneffekten die für die zweite Moderne so kennzeichnend sind. In Finanzmärkten begegnen Menschen seit jeher nicht einer dem individuellen Handeln äußeren >Natur< sondern sich selbst und ihren eigenen Reflexionen über die Welt der Finanzen. Das reflexive Element ist Finanzmärkten inhärent.

Gleichzeitig sind die vorherrschenden Denkmuster über Finanzmärkte nach wie vor von Ideen der ersten Moderne geprägt - vor allem in dem Teil der Wirtschaftswissenschaft, der auf Rationalismus und Utilitarismus, Universalität und Objektivität gründet. Im Gegensatz $\mathrm{zu}$ anderen Lebensbereichen sind die modernen Grundannahmen über unser Wirtschaftsleben und auch die Finanzmärkte in der gesellschaftlichen Praxis nie unterminiert worden. An Kritikern der orthodoxen Wirtschaftslehre hat es nicht gemangelt - man denke an Keynes, Mandelbrot oder den Ex-Weltbankdirektor Joseph Stiglitz. Die lange Tradition der Kritik allerdings macht die ungebrochene Autorität orthodoxen Denkens in der wirtschaftspolitischen Praxis nur noch bemerkenswerter (Généreux 2005). So wie Finanzmärkte selbst keine erste Moderne gekannt haben, ist in deren Reflexion die zweite Moderne noch nicht angebrochen.

Diese Anomalien sind aus zwei Perspektiven erklärungsbedürftig. Die Theorie der reflexiven Modernisierung kann uns auf der Suche nach den Ursachen des besonderen Verhaltens der Finanzmärkte helfen, während die Analyse von Finanzmärkten selbst ein neues Licht auf die Theorie der reflexiven Modernisierung wirft. Der erste Abschnitt geht den Fragen nach, die die Theorie der reflexiven Modernisierung aufwirft. Sie sieht das reflexive Element von Finanzmärkten und deren politischer Bearbeitung nicht als störendes Hintergrundrauschen, das es herauszufiltern gilt, will man zu > vernünftiger < Finanzmarktpolitik oder > validen $<$ wissenschaftlichen Ergebnissen kommen. Aus der Perspektive einer Theorie der reflexiven Modernisierung gilt das Gegenteil: Das reflexive Element wird zum Dreh- und Angelpunkt von Finanzmärkten, wie wir sie kennen, trotz - oder gerade wegen - der beharrlichen Versuche von Politik und Wissenschaft, dieser Reflexivität Herr zu werden. Damit wird die

* Ich danke Ulrich Beck, Thymian Bussemer, Peter Geschiere, Peter Pels und einem/einer Gutachter/ in der Sozialen Welt für hilfreiche Kommentare und Anregungen. 
Reflexion der Reflexivität in Finanzmärkten zur zentralen Frage: Wie gehen Politik und Wissenschaft mit dieser Reflexivität um, die Finanzmärkten von ihrem Entstehen an innewohnt? In den Worten von Beck und Holzer (2004, S. 169): »wann also kommt es zu [...] der Einsicht, dass man es statt mit Dingen an sich mit einer bloßen Externalisierung eigener Konstitutionsleistungen zu tun hat $[\ldots] \ll$ ?

Dass diese Einsicht in der Praxis bislang ausgeblieben ist, die Reflexivität also praktisch negiert wird, lässt sich mit Hilfe des von Latour (1993) geprägten Konzepts der >(diskursiven) Reinigung< [purification] verstehen. Diese >Reinigung< ist die Grundform modernen Denkens und Sprechens. Ihr liegt eine analytische Trennung der Dinge in einen transzendenten und einen immanenten Teil zugrunde, kurz gesagt also eine Aufspaltung der Welt in $>$ Natur $<$ (transzendent) und $>$ Kultur $</>$ das Soziale $<$ (immanent). Diese Denkfigur ist symptomatisch für die erste Moderne - und dominiert nach wie vor unsere Sicht auf Finanzmärkte. Letztere werden zerlegt in einen natürlichen, transzendenten Teil ( $>$ Marktkräfte $<)$ und einen sozialen, immanenten Teil (Finanzmarktpolitik im weiteren Sinne). Dieses analytische Raster, das der ersten Moderne geschuldet ist, steht damit in einem unaufgelösten Spannungsverhältnis zu der Reflexivität von Finanzmärkten selbst.

$\mathrm{Ab}$ dem zweiten Abschnitt wird diese >diskursive Reinigung < anhand der so genannten >Asienkrise< von 1997/98 illustriert. Obwohl die Finanzkrise viele >moderne< Annahmen über Finanzmärkte in Frage gestellt hat, ist es zu einer breitenwirksamen Reflexion der Reflexivität nicht gekommen. An ausgewählten Dokumenten, zum Beispiel vom Internationalen Währungsfonds (IWF), demonstriert der dritte Abschnitt die >diskursive Reinigung< von Finanzmärkten in der Praxis. Ein vierter Schritt zeigt, wie sich die Negation der Reflexivität in Finanzmarktpolitik niederschlägt - sowohl in der damaligen Reaktion auf die Asienkrise als auch in einer stets schwächeren Verankerung von Finanzmarktpolitik in demokratischen Prozessen, der Kehrseite einer steigenden Technokratisierung des Politikfeldes. Wie der fünfte Abschnitt dieses Artikels argumentiert, ist dieser Mangel an Reflektion genau in der Reflexivität von Finanzmärkten begründet. Die Selbstreferentialität der Marktgeschehens erfordert geradezu dessen Negation, um eine Implosion des Marktsystems zu verhindern. Der sechste Teil kehrt die Blickrichtung schließlich um: Was trägt das Beispiel der Finanzmärkte zu einer Theorie der reflexiven Modernisierung bei? Sind Finanzmärkte nur die Ausnahme, die die Regel bestätigen? Oder gibt es einen systematischen Zusammenhang zwischen der reflexiven Modernisierung anderer Gesellschaftsbereiche und dem scheinbaren Verharren der (Finanz-)Märkte in einer Negation der Reflexivität? Um es vorweg zu nehmen: Dieser Beitrag stützt letztere These. Zusammengefasst lautet sein Argument an diesem Punkt: Die reflexive Modernisierung nahezu aller Gesellschaftsbereiche wird erst dadurch möglich, dass sie einen >blinden Fleck $<$ hat $->$ Märkte $<$, und insbesondere Finanzmärkte als Ort abstrakter Quantifizierung von >Werten<. Marktlogiken werden mehr und mehr zum Anker zeitgenössischen Regierens, während die anderen Orientierungspunkte der ersten Moderne stets mehr im Nebel der Reflektion verschwimmen. ${ }^{1}$ Für die Theorie der reflexiven Modernisierung heißt das, dass gegenwärtige Entwicklungen der zweiten Moderne vielleicht mehr auf die >Anomalie< der (Finanz-)Märkte aufbauen als Kritikern der ersten Moderne recht sein kann. Zugespitzter formuliert: Vielleicht ist die >Vermarktung (oder Kommodifizierung) stets weiterer Teile der Gesellschaft eine notwendige Voraussetzung der zweiten Moderne.

1) Man denke zum Beispiel an Gesundheits-, Familien-, Sozial- und Bildungspolitik. Die heutzutage dominierenden Argumentationslinien in diesen Feldern sind in ihrem Kern ökonomisch und orientieren sich immer weniger an den Gesellschaftsbildern, Familienvorstellungen und Bildungsidealen der ersten Moderne. 


\section{Reflexivität und Reflexion in Finanzmärkten}

Auf den ersten Blick erfüllen globalisierte Finanzmärkte mehr als die meisten anderen Wirtschaftsbereiche die Grundbedingungen reflexiver Modernisierung (Beck/Bonß/Lau 2003). Sie sind, wie die Umweltgefahren denen Beck sich Mitte der 80er Jahre widmete, >historisches Produkt, [...] Spiegelbild menschlicher Handlungen und Unterlassungen, [und] Ausdruck hochentwickelter Produktivkräfte<(Beck 1986, S. 300). In Finanzmärkten begegnet der Mensch seit jeher sich selbst.

Dem >modernen< Menschen bereitet diese Selbstkonfrontation Unbehagen. Seit der kartesischen Differenzierung zwischen res cogitans und res extensa ist die Unterscheidung zwischen einer transzendent gedachten $>$ Natur $<$ auf der einen Seite und einem immanenten Bereich des Individuellen, Sozialen oder Gesellschaftlichen auf der anderen zur Grundfigur modernen Denkens geworden. Objekte, oder zumindest deren Bestandteile, werden durch oft mühevolle Gedankenakrobatik in eine der beiden Schubladen eingeordnet (Latour 1993). Krisen >modernen Denkens< haben immer wieder die Möglichkeit zu einer >reflektiven Wende < beinhaltet, wenn man die Reflexion der Reflexivität menschlichen Handels so nennen darf. ${ }^{2}$ Solche Krisen haben typischerweise eine Hinterfragung von Denkfiguren ermöglicht, die sich in letzter Instanz auf die vertrauensvolle Trennung unserer Welt in Natur und Gesellschaft, in Transzendenz und Immanenz stützen

Im Fall der Finanzmärkte ist vor allem die Reflektion der Reflexivität relevant, auch wenn die Theorie der reflexiven Modernisierung sich primär für die Reflexivität selbst interessiert. Erstens kann Reflexivität in den Finanzmärkten schwerlich als Lackmustest für den Übergang zwischen verschiedenen Phasen der Moderne dienen - sie ist ihnen schließlich inhärent. Zweitens aber ist die Reflexivität selbst eine Funktion von deren Reflektion - nun ganz im Gegensatz beispielsweise zur Umweltverschmutzung. Während die ökologischen Konsequenzen einer Abholzung des Regenwaldes in Endeffekt unabhängig von menschlichen Analyserastern sind, gibt es keine Finanzmärkte in denen deren Reflektion nicht schon enthalten wäre. Anders gesagt werden Finanzmärkte genau dann vollständig zu ihrem eigenen Gegenstand, wenn die prinzipielle Hoffnung auf externe Referenten (zum Beispiel ein $>$ messbares< Wirtschaftswachstum) und verlässliche Marktgesetzlichkeiten aufgegeben wird. Der entscheidende Punkt ist dann nicht, dass kritische Wissenschaftler diese Reflexivität schon lange reklamieren. Es interessiert an dieser Stelle auch (noch) nicht, dass ein Teil des Finanzmarktgeschehens sich mittlerweile darauf konzentriert, dessen innere Widersprüche gewinnbringend auszunutzen. ${ }^{3}$ Wichtig ist vielmehr, dass sich Finanzmarktpolitik und -praxis nach wie vor an einem >modernen< intellektuellen Rahmen orientieren der Marktgesetzlichkeiten und deren gesellschaftliche Verarbeitung klar scheidet.

2) Der Neologismus >reflektiv $<$ sei an dieser Stelle erlaubt, um die Unterscheidung zwischen Reflexivität und Reflexion (im Sinne von >Nachdenken über $<$ ) auch bei den Adjektiven beizubehalten zu können. Vor allem in der englischsprachigen Literatur haben Verwechslungen zwischen Reflexivität und Reflexion für reichlich Verwirrung gesorgt (vgl. Beck/Holzer 2004, S. 166ff). Wenn in diesem Beitrag >reflektiv < gebraucht wird, ist damit die Reflexion der Reflexivität von Finanzmärkten in Politik und Wissenschaft gemeint, also deren Anerkennung und Miteinbeziehung in Policy anstelle von deren Negation.

3) Eine stetige Steigerung der Selbstreferentialität des Finanzmarktgeschehens lässt sich durchaus beobachten. Aber dieser Vorgang ist graduell und entfaltet sich seit Jahrhunderten (Stringham 2003), auch wenn die technologischen Innovationen der letzten Dekaden die Möglichkeiten zur Verarbeitung dieser Selbstreferentialität vervielfacht haben (Partnoy 2002). Wir nehmen diesen Punkt an späterer Stelle wieder auf. Ich verdanke diesen Hinweis einem/r (anonymen) Gutachter/in des ursprünglichen Manuskripts. 
Im Bereich der > Wirtschaft $<$ findet sich die >moderne $<$ Denkfigur in der Idee des Marktes selbst - nicht im Sinne eines Marktplatzes, also einfach des Ortes oder der Gesamtheit von Tauschhandlungen - , sondern als normatives Konzept, durch das sich diese Tauschhandlungen an den von der neo-klassischen Wirtschaftslehre gestellten Bedingungen messen lassen müssen (Transparenz, Vertragsfreiheit, eindeutige Eigentumsrechte, etc.) (Généreux 2005). Wo diese Bedingungen erfüllt sind können >Marktkräfte< ihre Wirkung entfalten. Die Vorstellung von (nie direkt beobachtbaren) >Marktkräften< allerdings erlaubt eine spezielle analytische Operation: Mit ihr lässt sich wirtschaftliches Handeln im klassisch-soziologischen Sinn (wie zum Beispiel bei Weber) aufteilen in zwei grundverschiedene Zutaten: die Wirkung von Marktkräften und anderen, nicht-marktartigen Dynamiken. Adam Smith hatte die mittlerweile sprichwörtliche >unsichtbare Hand< noch ganz als Metapher für besondere empirische Beobachtungen gedacht. Mit dem >Mythos Markt(kraft)<(Engelen 1995) hingegen hat die neo-klassische Wirtschaftslehre der unsichtbaren Hand einen transzendenten Status zugewiesen:

[>The economy «] came to refer to a self-contained structure or mechanism whose internal parts are imagined to move in a dynamic and regular interaction, separate from the irregular interaction of the mechanism as a whole with what could now be called its exterior. A variety of other spaces could now be conceived in terms of their relationship to this hermetic field: the sphere of politics of the state; the sphere of law (previously at the center of questions of political economy); the sphere of science and technology; and the sphere of culture. (Mitchell 2002, S. 82)

Solange die (immanenten) Voraussetzungen stimmen und $>$ Marktkräfte $<$ nicht behindert werden, wirken sie durch ihre Verankerung in der menschlichen Natur. Die >Natürlichkeit< der Marktkräfte verweist automatisch alle Hindernisse zu ihrer Entfaltung in den Bereich des Sozialen.

Diese Denkfigur ist konstitutiv für die (neo-klassische) Wirtschaftswissenschaft. Sie begründet eine ontologische Unterscheidung zwischen >Markt< und >Staat $<$, die der ursprünglichen Vorstellung von >politischer Ö̈konomie< als geschicktem wirtschaftlichen (Regierungs-)Handeln noch völlig fremd war (Ibid., S. 80ff). ${ }^{4}$ Diese tief verwurzelte Dichotomie hat den Wirtschaftswissenschaften innerhalb der Sozialwissenschaften eine besondere Position verschafft: Im Gegensatz zu beinahe allen Konzepten, mit denen >moderne< Gesellschaften sich selbst beschrieben haben - Klasse, Rasse, Nation, Genderkategorien, Persönlichkeit, Gemeinschaft, Familie, Kultur - ist das Konzept $>$ Wirtschaft $<$ nie einer breitenwirksamen Kritik unterzogen worden (Ibid., S. 3). ${ }^{5}$ Die Modernisierung der ersten Moderne, die die zweite Moderne kennzeichnet, lässt im Fall der Wirtschaft nach wie vor auf sich warten.

Dabei ist der Hinweis auf die Immanenz der > Wirtschaft $<$ und die Reflexivität von Finanzmärkten natürlich alles andere als neu. Karl Polanyi (2001 [1944]) hat in seiner Great Transformation die Einbettung von Wirtschaftsgeschehen in breitere gesellschaftliche Prozesse eindrucksvoll beschrieben. Die institutionelle Komponente solchen Denkens ist in Klassikern der modernen Politischen Ökonomie wie Andrew Shonfield's Modern Capitalism (1965) oder John Zysmans Governments, Markets and Growth (1983) aufgenommen. Das Feld der >Socio-Economics $<$ hat die Verwobenheit des Wirtschaftslebens mit anderen sozia-

4) Marx ist einer der ersten gewesen, der die Reifizierung wirtschaftswissenschaftlicher Konzepte (allen voran natürlich des >Kapitals $<$ ) einer grundsätzlichen Kritik unterzogen hat. Gleichzeitig bleibt auch er dem Glauben an Gesetzmäßigkeiten im Wirtschaftsgeschehen verhaftet. Er entlarvt den Fetisch und die politische Ladung des Tauschwerts. Aber das rigide Theoriekonstrukt, das er an dessen Stelle setzt, basiert im Endeffekt ebenfalls auf objektiven Größen - diesmal dem Gebrauchswert von Dingen und der auf ihre Produktion verwendeten Arbeit. Seine geistesgeschichtliche Rolle im Hinblick auf die Reflexion der Reflexivität bleibt damit ambivalent.

5) Nicht zuletzt die zeitgenössische >Globalisierungskritik< verharrt in Denkmustern deren Überwindung eigentlich integraler (und wahrscheinlich unabdingbarer) Bestandteil ihres Projekts sein müsste. 
len Prozessen weiter ausgearbeitet (z.B. Granovetter 1985; Fligstein 2001; Swedberg 2003). Im Feld der Finanzmärkte stehen alle skeptischen Untersuchungen im Schatten von José de la Vegas Confusion de Confusiones aus 1688, einer akribischen Analyse des Amsterdamer Börsengeschehens der - von den Auswirkungen der Computerisierung einmal abgesehen bis heute wenig hinzuzufügen ist. ${ }^{6}$

Der Gegenentwurf zur neo-klassischen Sicht auf Finanzmärkte begriff letztere als selbstreferentielles System. In systemtheoretischer Perspektive zum Beispiel beziehen sich Risikoanalysen - Einschätzungen der Risiken, die in Finanzmärkten gehandelt werden - nicht auf objektiv gegebene, >externe< Referenten, sondern auf die Einschätzungen anderer Marktteilnehmer (Baekker 1991; 1999). >Erfolg < in Finanzmärkten wird damit losgekoppelt von >Fundamentaldaten<. Er basiert auf Vermutungen zweiten Grades: Vermutungen über die Vermutungen anderer Marktteilnehmer. Das aber heißt, dass Finanzmärkte Risiken nicht notwendigerweise zum Verschwinden bringen, sondern ebenso gut potenzieren können. ${ }^{7}$

Gerade weil diese Kritik Hunderte von Jahren alt ist, ist es erklärungsbedürftig, dass letztere sowohl im Großteil der Wirtschaftswissenschaften als auch der Finanzmarktpolitik so wenig Widerhall findet. Im Zuge der Verdrängung der Politischen Ökonomie durch die Wirtschaftswissenschaft im neunzehnten Jahrhundert schwang bei Debatten in Politik und Wissenschaft immer häufiger die implizite Frage mit, ob sich Finanzmärkte ihrer >Natur< nach als Gegenstand einer >exakten Wissenschaft< eigneten oder nicht. Und im Gegensatz zu anderen Bereichen sozialen Lebens ist diese Frage bis heute immer emphatisch mit ja beantwortet worden. Die oben genannten >reflektiven < Annäherungen an die Materie sind Ausnahmen geblieben. Aus einer, wie wir heute sagen würden, >qualitativen< Politischen Ökonomie - zum Beispiel dem so oft missverstandenen Adam Smith, aber auch Malthus, von Thünen, List, John Stuart Mill - wurde eine >quantitative Wissenschaft<.

In den 90er Jahren des zwanzigsten Jahrhunderts wurde >scientific finance< zu einer akademischen Boom-Industrie. Ihren Höhepunkt erreichte sie mit der Verleihung des Wirtschafts-Nobelpreises 1997 für die nach ihren Erfindern benannte Black-Scholes-Formel, die wissenschaftlich-exakte Berechnung von Finanzrisiken möglich machen sollte (de Goede 2001). In der Anwendung stellte sich diese Form von >scientific finance< schnell als >rocket science< heraus: Der Rolls Royce unter den Anlagefonds, Long Term Capital Management, der seinen Ruhm der Anwendung jener Formel verdankte, verlor im August 1998 an einem Tag \$550 Millionen und wurde nach einer von der amerikanischen Zentralbank ad hoc organisierten Notoperation abgewickelt (Dunbar 2000). Dem grundsätzlichen Glauben an die Möglichkeit wissenschaftlich-exakter Finanzanalyse konnte dieser Zusammenbruch allerdings keinen Abbruch tun. Die Chance zu einem solchen Paradigmenwechsel lag vielmehr in der zeitgleichen, wesentlich breiteren Diskussion um die Finanzkrise in Südostasien.

\section{Die Asienkrise als verpasste Chance einer Reflexion der Reflexivität in Finanzmärkten}

Finanzkrisen sind so alt wie Finanzmärkte selbst (Kindleberger 1978) - die an den Internetboom erinnernde Spekulation mit Tulpenknollen im Amsterdam des 17. Jahrhunderts ist nur eines der bizarreren Beispiele. Ebenso alt wie die Finanzkrisen ist die Einsicht, dass diese

6) Den Hinweis auf de la Vega verdanke ich dem ehemaligen Generalsekretär der Federation of European Securities Exchanges, Paul Arlman.

7) So ist der Mathematiker Benoit Mandelbrot, der Vater der fraktalen Geometrie und Wegbereiter mathematischer Ausarbeitungen der Chaostheorie, in seiner jüngsten Publikation zu dem Resultat gekommen, dass etablierte Finanzmarktmodelle inhärente Marktvolatilität und -risiken systematisch unterschätzen (Mandelbrot/Hudson 2004). 
den Stempel menschlicher Irrationalität, Hybris und Habgier tragen (Galbraith 1975) - kurz gesagt also (zumindest teilweise) das Produkt menschlicher Handlungen sind. Die Auswirkungen für die Betroffenen können verheerend sein. Der Wall Street-Crash von 1929 - mit all seinen Folgen in den USA und Europa - ist ein extremes Beispiel (James 2001), das aber die potentiellen Auswirkungen auf die >reale Wirtschaft< (also Konsum und Produktion) deutlich macht.

Auch die sogenannte Asienkrise von 1997/98 richtete in den am stärksten betroffenen Ländern - Thailand, Indonesien, Malaysia, den Philippinen und Süd-Korea - beträchtlichen wirtschaftlichen Schaden an (Knowles/Pernia/Racelis 1999). Die massiven Abwertungen der lokalen Währungen verteuerten nicht nur Importe sondern führten auch zu enormen Steigerungen der Lebensmittelpreise. Massive Zinserhöhungen - erfolglose Versuche den Kapitalabfluss zu bremsen - trieben viele Unternehmen in den Ruin und dämpften weitere Investitionen. Die realen Bruttoinlandsprodukte der genannten Staaten brachen teilweise um mehr als 10 Prozent ein (für eine Übersicht s. IWF 1998a, S. 46f).Die Krise hatte das Potential, zum Reflexions-Moment der Reflexivität in der globalen Finanzmarktordnung schlechthin zu werden und stellte somit den Lackmustest für die Reflexionsfähigkeit in der internationalen Finanzmarktpolitik dar. Zur Erinnerung:

Die Krise kam gänzlich unerwartet. Weder der Internationale Währungsfonds (IWF) noch die Mehrheit der in der Region aktiven Investmentbanken hatten die Krise vorhergesehen; letztere hatten bis kurz vor Beginn der Krise Kapital nach Südostasien geleitet. Die südostasiatischen Tiger galten als vorbildlich in ihrer >aufholenden Entwicklung . Die Schuldenniveaus waren nicht überdurchschnittlich und die Volkswirtschaften verzeichneten hohe Wachstumsraten. Verglichen mit dem Rest der Welt sah die wirtschaftliche Situation in Südostasien Mitte der 90er Jahre solide und vielversprechend aus. Und doch leitete die Abwertung des thailändischen Baht durch die Regierung in Bangkok am 2. Juli 1997 eine die ganze Region erfassende monatelange Kapitalflucht ein, die durch Anpassung der Finanzmärkte an >fundamentale Wirtschaftsdaten< schwerlich nicht zu erklären war. Was war passiert?

$>$ Globalisierungskritische< Stimmen machten entfesselten >Kasino-Kapitalismus $<$ (Strange 1986; 1998) für die Krise verantwortlich und nahmen vor allem den IWF ins Visier (Crotty/ Dymski 1998; Feldstein 1998; Sachs/Radelet 1998; Feldstein 1999; Krugman 2000) . Die Kritik an letzterem hatte drei Hauptkomponenten: Erstens habe er im Vorfeld der Krise mit einer einseitigen Marktorthodoxie ein Politikprogramm gepredigt, das die enormen Kapitalzu- und abflüsse überhaupt ermöglicht habe. Zweitens habe er als Hüter des Weltfinanzsystems die Krise vorhersehen oder zumindest eine plausible Erklärung für die Krise parat haben müssen, als deren Ausmaß deutlich wurde. Beides war nicht der Fall. Drittens wurde die Antwort des IWFs auf die Krise - vor allem die strengen Auflagen, an die Notkredite geknüpft wurden - als nicht nur uneffektiv, sondern geradezu kontraproduktiv gescholten.

Ein Großteil der Kritiker stellte implizit die >Reinheit< von Marktkräften in Frage (Armijo 2001). Real existierende Finanzmärkte, so zum Beispiel der Tenor von Jeffrey Sachs und Steven Radelet (1998), hätten eine inhärente Neigung zu Volatilität. Der Kern dessen, was zur Debatte stand, war die Validität der Unterscheidung zwischen einem objektiv gegebenen Risiko einerseits und dessen (mehr oder weniger zutreffender) Analyse durch Finanzmarktteilnehmer andererseits. Die Einschätzung des Investors George Soros steht stellvertretend für eine kritische Einschätzung:

Immer wieder wird behauptet, ökonomische Angelegenheiten seien unabänderlichen Regeln unterworfen, vergleichbar den Gesetzen der Physik. Das ist falsch. Mehr noch: Entscheidungen und Strukturen, die sich auf diese Überzeugung stützen, wirken wirtschaftlich destabilisierend und sind politisch ge- 
fährlich. Denn das Marktsystem ist, wie jedes andere menschliche Konstrukt, seinem Wesen nach mangelhaft [...]. (Soros 1998, S. 61)

Was den meisten Beobachtern überdies fehle, so Soros, sei die Einsicht in das, was er selbst $>$ Reflexivität< nennt: >Es gibt keine vom Denken der [an Finanzmärkten] Beteiligten unabhängige Realität, sie ist mit ihren Reflexionen eng verwoben.<(Ibid., S. 83) In einem solchen selbstreferentiellen System der Preisbildung verschwimmt die Grenze zwischen Objektivität und Subjektivität und damit die Unterscheidung zwischen (der prinzipiellen Möglichkeit zu) >reinen< Marktkräften und deren Verzerrung durch gesellschaftliche und politische Umstände. In der Asienkrise war damit eine Diskussion um die Grundannahmen der neoklassischen Wirtschaftswissenschaftlichen angelegt, auf denen nach wie vor der Großteil von Politik in diesem Bereich fußte.

\section{Die >diskursive Reinigung « von Marktkräften}

Dass es trotz aller Kritik zu einer Reflexion der Reflexivität in der globalen Finanzmarktpolitik nicht gekommen ist, lässt sich nur mit Blick auf die oberflächlich erfolgreiche Bearbeitung des Problems mittels der Technik der >diskursive Reinigung<verstehen. Diese >Reinigung < darf dabei nicht als pure Ideologie oder eine Vertuschung von Marktunzulänglichkeiten missverstanden werden. Sie beruht darauf, dass die neo-klassische >Sprache< auch durch die Asienkrise nicht an ihre Grenzen gebracht wurde. Die für sie konstitutive Unterscheidung zwischen transzendenten Marktkräften und immanenter Politik funktionierte als diskursives Sicherheitsventil eines >Sprachspiels< (Rorty 1992) das durch die Krise mehr denn je unter Druck geraten war.

Die diskursive Reinigung lässt sich beispielhaft an zwei Typen von Analysen illustrieren: Zum einen an zentralen Dokumenten des IWF wie The IMF's Response to the Asian Crisis (IWF 1999) und The Asian Crisis: Causes and Cures (IWF 1998b) und zum anderen an unabhängigen wissenschaftlichen Analysen, die der IWF-Linie aber im großen und ganzen folgen (Corsetti/Pesenti/Roubini 1998; Frankel 1998). Der zentrale Punkt dabei ist die Naturalisierung von Marktkräften und der daraus folgende Verweis von >Politik < und >Kultur< (als Gegenkonzepten) in den Bereich des Sozialen und Immanenten.

Der erste Schritt der Aufarbeitung liegt in der prinzipiellen Aufrechterhaltung der analytischen Trennung zwischen einem objektiv gegebenen Marktrisiko und dessen subjektiver Einschätzung durch Marktteilnehmer - eine Unterscheidung, die der Vorstellung von Finanzmärkten als selbstreferentiellem System von Einschätzungen von Einschätzungen direkt zuwiderläuft. Michel Camdessus, seinerzeit Managing Director des IWF, hat die Situation wie folgt beschrieben:

In many respects, this deepening of the crisis in some countries and its contagion to others reflects $r a-$ tional market behavior. But certainly the amount of exchange rate adjustment of the Thai baht, the Indonesian rupiah, and the Korean won, among other currencies, far exceeds any reasonable estimate of what might have been required to correct their initial overvaluation. In this respect, the markets have overreacted. (Camdessus 1998; Hervorhebungen vom Autor)

Marktteilnehmer sind also von dem, was die Marktsituation eigentlich gefordert hätte, in ihrer Bewertung abgewichen. Das Problem waren nicht Marktkräfte selbst, sondern das >poor assessment and management of financial risk< (IWF 1999), also Fehleinschätzungen im Prinzip objektiv gegebener Risiken: >international investors had underestimated the risks as they searched for higher yields (IWF 1998b; Hervorhebung vom Autor). Aber wie lassen sich diese Fehleinschätzungen >sauber erklären - auf eine Weise, die die Unterscheidung zwischen objektivem Risiko und subjektiver Einschätzung aufrecht erhält? Zumindest zum Teil, so der IWF, sind sie zurückzuführen auf >problems resulting from the limited availability of data and a lack of transparency, both of which hindered market participants from 
taking a realistic view of economic fundamentals (IWF 1998b; Hervorhebung vom Autor). Die Ursache lag also in praktischen Problemen, die Marktteilnehmern die Sicht verstellten und an der Ausübung ihrer >Funktion< gehindert haben.

In dieser Sicht wird die moderne Unterscheidung zwischen transzendenten Marktkräften und immanenter Policy und Politik radikalisiert. Erst die universalistisch gedachte Geltungsmacht von Marktgesetzlichkeiten erlaubt es, den von der Krise betroffenen Ländern >inadequate financial sector supervision< (IWF 1999; Hervorhebung vom Autor) und > [a] lack of enforcement of prudential rules< (IWF 1998b) zu diagnostizieren. Die >Unangemessenheit< von Public Policy bezieht sich auf das Unvermögen, immanente Politik an transzendente Marktgesetzlichkeiten anzupassen. Letztere bringen objektiv feststellbare Ansprüche an Public Policy mit sich: die Schaffung der institutionellen Voraussetzung, unter denen Marktkräfte unverzerrt zum Tragen kommen können. In diesem Punkt aber haben die Regierungen der betroffenen Länder versagt:

Partly because of the large-scale financial inflows that their economic success encouraged, there were also increased demands on policies and institutions, especially those safeguarding the financial sector; and policies and institutions failed to keep pace with these demands [...]. Only as the crisis deepened were the fundamental policy shortcomings and their ramifications fully revealed. (IWF 1998b; Hervorhebungen vom Autor)

Das Unvermögen geht allerdings noch weiter:

Central to a full understanding of the roots of the Asian crisis is the multifaceted evidence on the structure of incentives under which the corporate and financial sectors operated in the region, in the context of regulatory inadequacies and close links between public and private institutions. (Corsetti/Pesenti/ Roubini 1998, Hervorhebungen vom Autor)

Erstens haben - so die gängige Argumentation - (politisch geschaffene) Anreizstrukturen >natürliche< Marktdynamiken verzerrt. Zweitens aber haben die Regierungen in der Region durch enge Beziehungen zwischen öffentlichen und privaten Institutionen der (unterstellten) ontologischen Trennung zwischen >Markt< und >Staat< unzureichend Rechnung getragen bzw. diese systematisch durchbrochen.

Die transzendente Qualität von Marktgesetzlichkeiten impliziert deren universale Geltung - und damit die Legitimität einer global einheitlichen Finanzmarktpolicy, begründet auf Expertise. Jeffrey Frankel, Harvard Professor, ehemaliger Berater des IWF und seinerzeit Mitglied im Council of Economic Advisers des amerikanischen Präsidenten, formulierte es 1998 in einer Rede vor der US International Trade Commission wie folgt:

The Asian style of corporate governance tends in the direction of empire-building, that is, maximizing market share, rather than what neoclassical economic theory says firms should maximize, namely profitability or the price of the company's stock. Shareholders and consumers lost out. For a while it looked like this was an arcane theoretical point, of interest to economists but not to real-world firms. How could there ever be too much investment or too much growth? Now we see that Asian firms made precisely this mistake. They developed excess capacity in such sectors as steel and electronics, and are now paying the price. This is what I had in mind when I said that the rules of economics turn out to apply to East Asia similarly to elsewhere. (Frankel 1998, S. 2, Hervorhebungen vom Autor)

Der angedeutete kulturalistische Twist wird im Folgenden noch deutlicher:

One should include on the list of Asian economic flaws: patterns of corruption, industrial policy and other excessive government interference in the economy. I will even venture to cross over from economics to politics [...]. We were told in the past that Asian values did not place as high weight as did Westerners on democracy, free speech and other civil freedoms. I think many Asians may have concluded recently - even leaving aside the non-economic benefits of such rights - that there are financial 
advantages to the rule of law, transparency, freedom of expression, and clearly-established procedures for government succession. (Ibid., S. 3, Hervorhebung vom Autor)

Zynisch gesehen wird die Finanzkrise damit zur Rächerin für die Missachtung universell geltender Werte.

Die hier angeführten Zitate stehen beispielhaft für die gelungene diskursive Reinigung von >transzendenten Marktkräften < - einer Denkfigur die durch die Asienkrise in einer bis dato nicht gekannten Weise auf die Probe gestellt wurde. Gleichzeitig, auch das zeigen die Zitate, ist es möglich geblieben, die Krise und ihre Ursachen in einer Sprache zu beschreiben, die die Trennung zwischen Marktkräften und ihrer immanenten Umgebung aufrechterhält. Ironischerweise basiert diese Möglichkeit auf der >Unreinheit< unserer ökonomischen Wirklichkeit selbst. >Marktkräfte< entfalten sich nur in realen, sozialen Kontexten; die Möglichkeit, sie in natura zu beobachten ist damit von vornherein ausgeschlossen. Das bedeutet aber auch, das die Zuschreibung von >Marktversagen<, zum Beispiel einer Finanzkrise, arbiträr bleibt. Anders gesagt, es bleibt immer möglich, die Ursachen von Marktversagen der $>$ Verunreinigung< von Marktkräften zuzuschreiben. Damit ist das Wirtschaftssystem nicht vor realen Krisen geschützt, wohl aber vor externer Kritik an der Vorstellung von im Prinzip regelmäßigen Marktkräften. Dieser Effekt potenziert sich mit der wachsenden Komplexität unseres Wirtschaftssystems, da stets mehr immanente Faktoren als Erklärungen für Marktversagen herangezogen werden können.

\section{Die Reaktion der >Internationalen Gemeinschaft}

Aber welche Konsequenzen hat eine solche diskursive Verarbeitung von zum Beispiel der Asienkrise für die politische Praxis gehabt? Handelt es sich bei diesen Denkmustern um mehr als reine $>$ Sprachspiele $<$ ? Bemerkenswert an der Reaktion der >internationalen Gemeinschaft $<$ ist zunächst, welche Konsequenzen aus der Asienkrise nicht gezogen wurden. Die wieder und wieder diskutierte Tobin-Steuer zum Beispiel - gedacht als >Sand im Getriebe der globalen Finanzmärkte $<$ - wurde auf internationaler Ebene nicht in Erwägung gezogen. Zweifel an ihrem letztlichen Nutzen sind berechtigt. Trotzdem hätte die Tobinsteuer als ernstgenommener Vorschlag einen bemerkenswerten Wechsel der Denkrichtung markieren können. Erstens hätte sie eine Erweiterung des Diskurses um Fragen dargestellt, die auf Werte verweisen, welche außerhalb der Finanzmärkte selbst liegen (z.B. Gerechtigkeit oder selbstbestimmte Entwicklung im Gegensatz zu allokativer Effizienz). Damit wäre die Idee eingeführt worden, dass globale Finanzmarktpolitik andere Ziele haben könnte als die Optimierung des operativen Umfelds von Marktakteuren selbst, auch wenn eine solche Optimierung durchaus mit dem Ziel allgemeiner Wohlfahrtssteigerungen propagiert wird. Zweitens hätte sie einen Bruch mit dem Glauben an diese Optimierbarkeit selbst dargestellt und somit einen Blick auf die Frage eröffnet, ob Finanzmärkte vielleicht vor den ihnen inhärenten Kräften geschützt werden müssen. Das Entscheidende wäre dann nicht Sinn oder Unsinn der Tobin-Steuer selbst gewesen, sondern die Erweiterung des diskursiven Möglichkeitsraums, in dem Finanzmärkte gesellschaftlich und politisch bearbeitet werden.

In der Tat wurde die >globale Finanzarchitektur < durch die Asienkrise zum Gegenstand umfassender Diskussionen auf höchster Ebene (Council on Foreign Relations Independent Task Force 1999; Eichengreen 1999). Eine Störung des >Policy-Gleichgewichts< (True/ Jones/Baumgartner 1999) schaffte also die Möglichkeit zu einem Paradigmenwechsel (Hall 1993) und einer grundsätzlichen politischen Neuorientierung. Allein, bei ihrem Treffen in Köln im Juni 1999, auf dem die >Asienkrise< und die zu ziehenden Konsequenzen ganz oben auf der Agenda standen, folgten die G7-Finanzminister der oben dargelegten orthodoxen Sichtweise der Krise und deren impliziten Grundannahmen: 
Our overall strategy is to identify and put in place policies to help markets work properly and to provide the public goods necessary to achieve this objective. This requires public authorities to provide for enhanced transparency and disclosure, improved regulation and supervision of financial institutions and markets, and policies to protect the most vulnerable. It also requires that private creditors and investors bear responsibility for the risks that they take, and are involved appropriately in crisis prevention and crisis management. In these respects, the establishment of internationally-agreed codes and standards for policy-makers serves both as an incentive for better governance and as a yardstick against which to measure country risk. (G7 Finance Ministers 1999, Hervorhebungen vom Autor)

Im Kern soll also immanente Policy die >ordentliche< Funktion von Märkten gewährleisten. Zur Erreichung dieses Ziels, so der Entschluss, müssten Standards und Codes erstellt werden, an denen sich nationale Regelungen messen lassen müssen.

Im selben Jahr noch ist auf Initiative der G7 das >Financial Stability Forum< (FSF) gegründet und mit dem Mandat ausgestattet worden, ein Kompendium von zwölf Standards und Codes zusammenzustellen, das die ganze Bandbreite von >Finanzmarktgovernance< abdeckt. ${ }^{8}$ Die Überprüfung der Implementation dieser Standards und Codes ist dem IWF übertragen. Letzterer veröffentlicht regelmäßig >Reports on the Observance of Standards and Codes < für dessen Mitgliedsstaaten und führt >Financial Sector Assessment Programs < durch (Soederberg 2002, S. 615ff). Die Ergebnisse dieser Überprüfungen wiederum sollen die großen Rating-Agenturen bei ihren Schätzungen von länderspezifischen Risiken in Betracht ziehen (Strulik 2000). Diese Ratings bilden unter den von dem Basler Komitee für Bankenaufsicht erstellten neuen Regeln (bekannt als Basel II) die Grundlage der Höhe der verpflichtenden Kapitalrücklagen, mit denen Kreditinstitute sich intern gegen mögliche Bankrotte ihrer Schuldner versichern müssen; damit bilden sie die Grunddeterminante der Zinsen, die einzelne Schuldner für Kredite zahlen müssen. In der Theorie der neuen Finanzarchitektur werden Zinsen für Kredite an `Entwicklungsländer $<$ - um letztere geht es ja im Kern - zum Instrument der Durchsetzung universal geltender Standards und Codes.

Das Design der Standards, aus denen das Kompendium des FSF besteht, obliegt spezialisierten Expertenorganisationen, die von effektiver demokratischer Kontrolle weit entfernt sind. Die Diskussion um eine Regulierung von Rating-Agenturen gibt hierfür ein anschauliches Beispiel ab. Die beiden größten unter ihnen, Standard \& Poor's und Moody's, hatten 2003 die Kreditwürdigkeit einer Reihe großer deutscher Unternehmen teilweise bis auf $>$ Junk<-Status herabgestuft und damit automatisch deren Kapitalkosten erhöht (Financial Times 2004). Diese Unternehmen - unter ihnen vor allem Thyssen-Krupp - fühlten sich ungerecht behandelt und unterstellten den Rating-Agenturen, angloamerikanische Methoden der Unternehmensführung in der Bewertung von Kreditwürdigkeit prinzipiell und ohne Rücksichtnahme auf nationale Standorte einseitig zu bevorzugen. Egal ob zu recht oder nicht weckten diese Vorfälle in der deutschen Politik den Ruf nach staatlicher Regulierung von

8) Diese Standards umfassen den >Code of Good Practices on Transparency in Monetary and Financial Policies $<$ (IWF), den >Code of Good Practices on Fiscal Transparency $<$ (IWF), den >Special Data Dissemination Standard < und >General Data Dissemination Standard ( (beide IWF), >Principles and Guidelines for Effective Insolvency and Creditor Rights Systems< (koordiniert durch die Weltbank), die $>$ Principles of Corporate Governance $<(\mathrm{OECD})$, die >International Accounting Standards $<$ (International Accounting Standards Board), die >International Standards on Auditing $<$ (International Auditing and Assurance Standards Board), die >Core Principles for Systemically Important Payment Systems (Committee on Payment and Settlement Systems), die >Forty Recommendations of the Financial Action Task Force on Money Laundering< (Financial Action Task Force), die >Core Principles for Effective Banking Supervision< (Basler Kommitee für Bankenaufsicht), die >Objectives and Principles of Securities Regulation < (International Organisation of Securities Commissions), und die >Insurance Core Principles (International Association of Insurance Supervisors). (Die Titel der Standards und Codes sowie der für sie verantwortlichen Organisationen sind großenteils nicht übersetzt da auch im deutschen Sprachraum deren englische Namen gängig sind.) 
Rating-Agenturen (Handelsblatt 2004). De facto war diese Diskussion allerdings eine Scheindebatte. Die eigentliche Entscheidung über eine öffentliche Regulierung von RatingAgenturen wurde zwar in Berlin getroffen, aber nicht etwa durch den Bundestag oder das Finanzministerium, sondern durch die International Organisation of Securities Commissions (IOSCO), also den Zusammenschluss nationaler Wertpapiermarktregulierungsbehörden mit eigentlichem Sitz in Madrid, deren Task Force zu Rating-Agenturen zur Verkündung ihrer Ergebnisse in der deutschen Hauptstadt weilte (IOSCO Pressemitteilung vom 3. Dezember 2004). Der Kern dieser Entscheidung war, von direkter Regulierung abzusehen und auf $>$ Marktdisziplin< zu setzen. Rating-Agenturen sollen in Zukunft öffentlich machen, wie sie mit den Geboten eines neu erstellten >Code of Conduct< umgehen, so dass andere Marktteilnehmer sich selbst ein Bild vom internen Management dieser Agenturen machen können (Financial Times 2004). Der zentrale Punkt ist an dieser Stelle nicht so sehr der Inhalt der Entscheidung, sondern deren Zustandekommen. Neben von genuin politischen Organen relativ unabhängigen Regulierungsbehörden waren es vor allem Finanzmarktakteure sowie deren Interessenorganisationen, die sich in der Konsultationsrunde aktiv in IOSCOs Entscheidungsfindung einbringen konnten. Der Vorsitzende der Task Force hob dementsprechend hervor, dass die Beteiligung der >Öffentlichkeit< (in der Form von 39 schriftlichen Antworten in der Konsultationsrunde, abrufbar über IOSCOs Website) entscheidend zum Erfolg der Task Force beigetragen habe und letztere damit zu einem Modell für zukünftige Arbeit von IOSCO werde (IOSCO Pressemitteilung vom 3. Dezember 2004).

Freilich, in der politischen Bewertung solcher Verfahren treffen Welten aufeinander. Aus demokratietheoretischer Sicht wirft die Technokratisierung von Finanzmarktpolitik eindeutige Probleme auf (Germain 2001; Underhill 2001; Pauly 2002). Von >innen< betrachtet hingegen - also aus der Perspektive der Finanzmärkte selbst - ist eine De-Politisierung von Finanzmarktregulierung nicht nur legitim (da sie, richtig institutionalisiert, den Policy-Output erhöhen soll), sondern auch die einzige Chance, ein >funktionierendes globales Finanzmarktregime aufzubauen. Die Komplexität der Materie und die Innovationsgeschwindigkeit der Finanzmärkte selbst machen jegliche >Hoffnung < auf eine breite, öffentliche Deliberation schnell zunichte. Kontur wie Lösung des Problems hängen also ganz von der Perspektive ab.

Mit der Frage nach der Perspektive aber kehren wir zum Ausgangspunkt zurück: Wie kommt es, dass in den Finanzmärkten - ganz im Gegensatz zu anderen Gesellschaftsbereichen - eine zweite Moderne in Sinne einer kritischen Hinterfragung (erster) >moderner< Grundannahmen nicht stattgefunden hat? Populistische Perspektiven sehen in der Kritikresistenz der Marktorthodoxie wenig mehr als den Unwillen einer herrschenden Finanzelite, anderen gesellschaftlichen Gruppen Teilhabe an politischen Entscheidungsprozessen und damit den unzweifelhaften materiellen Segnungen, die Finanzmärkte bereithalten, zu gewähren. Aus einem wissenschaftlicher Blickwinkel kann das kaum befriedigen. In zwei letzten Schritten soll daher versucht werden, zumindest den Anfang einer Antwort auf diese Frage zu geben. Sie gründet erstens auf dem besonderen Charakter von Finanzmärkten selbst, zweitens aber auf der Position von Finanzmärkten als Ort quantitativer Wertmessung in der >zweiten Moderne< allgemein. Dieser letzte Schritt wirft dann so ein neues Licht auf die Theorie reflexiver Modernisierung selbst.

\section{Finanzmärkte und die Theorie reflexiver Modernisierung}

Wie oben gezeigt sperren sich Finanzmärkte dem Analyseraster der Theorie der reflexiven Modernisierung durch das untypische Verhältnis von Reflexivität und deren Reflektion. In den meisten anderen Gesellschaftsbereichen können wir uns die Entwicklung in Stufen denken: erst die traditionelle Gesellschaft, dann die erste Moderne, dann die zweite Moderne (hier kommt Reflexivität ins Spiel), und zuletzt, hoffentlich, eine weitere Stufe, in der die 
Reflexivität gesellschaftlich reflektiert wird. Die Möglichkeit dieser letzten Stufe - der Reflektion von Reflexivität - beruht aber darauf, dass es eine erste Moderne gegeben hat. Die Anerkennung der Reflexivität führt nur dann nicht zu einem Kurzschluss des Systems wenn sie nicht alle Orientierungspunkte ausradiert. Reflektion ist also nur in einem nicht vollständig geschlossenem System möglich; es müssen externe Referenzpunkte vorhanden sein, damit in der Analyse überhaupt noch etwas übrig bleibt, nachdem die Selbstreferentialität einmal anerkannt ist. Diese Bedingung ist in Finanzmärkten nicht erfüllt. Die Vorstellung von regelmäßigen Marktkräften bilden das Fundament für den Glauben, dass Finanzmärkte in einem geordneten Verhältnis zur >realen Wirtschaft< stehen - man erinnere sich an dieser Stelle noch einmal an den Verweis des IWF auf die >Fehlinterpretation< von wirtschaftlichen $>$ Fundamentaldaten $<$ als Faktor in der Asienkrise.

Dabei wird in der Praxis die Koppelung des Finanzmarktgeschehens an solche $>$ Fundamentaldaten immer schwächer. Entgegen der Erwartungen beinahe aller Beobachter und im Widerspruch zu gängigen Finanzmarkttheorien haben zum Beispiel die rasant steigenden amerikanischen Haushalts- und Zahlungsbilanzdefizite bislang nicht zu einem Einbruch des US Dollars geführt. Seit den computergestützten Innovationen der 1980er Jahre wachsen die sogenannten Derivatmärkte ungebremst (Partnoy 2002). Desto komplexer und unübersichtlicher aber die gehandelten Produkte werden, desto wichtiger wird das grundsätzliche Vertrauen in eine >unsichtbare Hand<, die die gegenwärtigen Entwicklungen doch noch einem guten Ende entgegenführt und auf dem Weg dahin für die >notwendigen < Korrekturen sorgt.

Im Endeffekt beruht die Selbstreferentialität von Finanzmärkten auf der Unmöglichkeit objektiver Wertmessung (Généreux 2005, S. 55 ff). Sie ist damit ein Phänomen, das das ganze Wirtschaftssystem umfasst. Ricardo und Marx waren sich dieses Problems gleichermaßen bewusst - und sind beide an ihm gescheitert. In der Folge hat die Neo-Klassik es schlicht ignoriert mit dem Axiom, dass >der Markt< selbst den Wert von Gütern, Rohstoffen, Arbeit und Dienstleistungen verlässlich angeben könne, dass also keine selbständige Werttheorie brauche wer eine gute Markttheorie habe. Dieses Postulat ist auf den zweiten Blick so fehlerhaft wie es auf der ersten Blick einleuchtet. ${ }^{9}$ Aber auch außerhalb akademischer Dispute stoßen >Messungen< von Wirtschaftsgrößen regelmäßig an ihre Grenzen. Der arbiträre Charakter von Messverfahren für Inflation, Arbeitslosigkeit, dem Bruttoinlandsprodukt, Produktivitätssteigerungen, Haushaltsdefiziten usw. steht im Kontrast zu deren stets steigender Wirkungsmacht im politischen Tagesgeschäft.

Die Negation dieser Probleme in der gesellschaftlichen Praxis fußt auf dem Mangel an konzeptionellen Alternativen. Die Anerkennung der Selbstreferentialität von Wertmessungen im Wirtschaftsgeschehen würde zu derartigen >Kurzschlüssen< im System führen, dass sie - durchaus im wörtlichen Sinn - kaum denkbar ist. Durch welchen Wertekanon sollte ein markt-basiertes System ersetzt werden, vor allem wenn es um die Organisation unseres wirtschaftlichen Lebens geht? Angesichts der Bedeutung und Komplexität, die sie heute haben,

9) Es würde wiederum zu weit führen, die Problematik umfassend zu erörtern. Sie lässt sich folgendermaßen zusammenfassen: Der >Marktwert < zum Beispiel von Arbeit oder Investitionsgütern hängt davon ab, welchen >Mehrwert< ein Unternehmen glaubt mit ihnen produzieren zu können. Der aber ist eine arbiträre Größe, die sich in letzter Instanz an Kapitalkosten orientiert (also den Zinsen die Unternehmen für die Anschaffung von Investitionsgütern bezahlen). Und diese Zinsen wiederum sind eine Funktion des erwarteten Mehrwerts von Investitionen, der rein davon abhängt, wie viel des Endproduktes dem Faktor Arbeit und wie viel dem Faktor Kapital zugeschrieben wird. So lässt sich kurz eine der beiden Positionen in den sogenannten Cambridge-Kontroversen der 1960er Jahre zusammenfassen, in denen Wirtschaftswissenschaftler der objektiven Basis von Kapitalkosten auf den Grund gehen wollten. Für die Anhänger der Neo-Klassik stellte sich das Problem als so grundlegend heraus, dass es seitdem weitgehend ignoriert wird. Für einen Einstieg in die Problematik s. Nitzan (1998, S. 171ff). 
sind (Finanz-)Märkte zum Erfolg verdammt, ungeachtet der Gedankenakrobatik, statistischen Salti und ständigen >diskursiven Reinigung < die dazu erforderlich sind.

\section{Finanzmärkte als Fels in der Brandung der zweiten Moderne}

Vielleicht liegt das Problem der >Finanzmärkte als Anomalie in einer Theorie der reflexiven Modernisierung d daher eher in der Fragestellung selbst. Sie hat sich aus der Vermutung ergeben, dass >reflexive Modernisierung < Gesellschaften in ihrer gesamten >Breite< erfasse und wir daher mehr oder weniger parallele Entwicklungen quer durch verschiedene soziale Bereiche erwarten sollten. Gesellschaften durchlaufen >Stufen < - von der traditionellen Gesellschaft über die erste zur zweiten Moderne. Nun besteht aber, auch für die Theorie der reflexiven Modernisierung, ein grundsätzlicher Unterschied zwischen dem ersten Übergang (von traditioneller Gesellschaft zu erster Moderne) und dem zweiten (von erster zu zweiter Moderne). Die Kritik an der traditionellen Gesellschaft kennt einen eindeutig definierten Ausgangspunkt: >moderne Überzeugungen $<$. Sie machen soziale Veränderungen möglich und halten gleichzeitig Orientierungspunkte bereit. ${ }^{10}$ Das ist in der zweiten Moderne anders.

Beck et al. (2003) haben wiederholt klargestellt, dass sie die Theorie der reflexiven Modernisierung durchaus in Opposition zu >postmodernen< Theorien verstanden wissen wollen, Theorien also, die gegenwärtigen Gesellschaften die Auflösung bis dato stabiler Wertsysteme diagnostizieren. Dem ist prinzipiell zuzustimmen. Damit ist allerdings noch nicht geklärt, wodurch die Wertesysteme der ersten Moderne, die dem zweiten Modernisierungsschub zum Opfer fallen, ersetzt werden. An diesem Punkt erlangen Märkte - und insbesondere Finanzmärkte - ihre besondere Bedeutung in der zweiten Moderne. Ist es nicht so, dass in stets mehr Bereichen gesellschaftlichen Lebens die Wertlogiken der ersten Moderne durch Marktlogiken ersetzt werden? Anders gefragt: Hängt nicht vielleicht die zweite Moderne als Phänomen davon ab, dass >der Markt< Orientierungshilfen bereithält, sobald wir uns von modernen Leitbildern verabschiedet haben?

Es würde zu weit führen, diese These an dieser Stelle vollständig auszuführen. Es bleibt aber festzuhalten, dass die Erosion >moderner Werte (die mit der ersten, mittlerweile auch schon >traditionelle< Moderne assoziiert werden) Hand in Hand geht mit dem Aufstieg marktorientierter Werte. Der Nationalstaat, basierend auf einer ehedem homogen gedachten Nation als Solidargemeinschaft, macht dem Wettbewerbsstaat Platz (Cerny 1990). In dem $\mathrm{Maße}$, in dem wir unseren Glauben an Kultur (mit einem großen $>\mathrm{K} \prec$ ) verloren haben, ist im kulturellen Leben Raum für dessen Kommerzialisierung entstanden. Die Liste ließe sich beliebig fortsetzen.

Vielleicht hält die Synthese des >Sonderfalls` Finanzmärkte und des von der Theorie der reflexiven Modernisierung diagnostizierten generellen Übergangs zu einer >zweiten Moderne damit die Erklärung für die scheinbar unaufhaltbare Kommodifizierung von stets mehr Gesellschaftsbereichen bereit. Das reflexive Element der zweiten Moderne führt etablierte Denkmuster an ihre Grenzen und trägt damit in vielen Bereichen zu einer Reflektion der Reflexivität bei. Tradierte Wertesysteme brechen auf. Allein (Finanz-)Märkte sperren sich - wie oben dargelegt - einer solchen Reflektion. Unfreiwillig stehen sie wie ein Fels in der Brandung wachsender Unsicherheiten und halten Orientierungspotential bereit für all die Gesellschaftsbereiche, deren angestammte Wertsysteme der Reflektion von Reflexivität zum

10) Wie Karl Polanyi gezeigt hat hieß das keineswegs, dass die sozialen Umwälzungen dadurch vernachlässigbar geworden wären. 
Opfer gefallen sind. ${ }^{11} \mathrm{Da}$ sich aber gesellschaftliche und politische Praxis zunehmend an Marktkategorien orientiert (zum Beispiel Effizienz oder Produktivität), wird das Vertrauen in die Möglichkeit einer Trennung zwischen transzendenten >Marktkräften< und deren immanenter sozialer Umgebung nur noch größer. Zynisch formuliert ist es vielleicht die Unfähigkeit des Marktsystems zur Selbstreflektion, auf der seine stets steigende gesellschaftliche Hegemonie begründet ist.

\section{Schlussbetrachtungen}

Marx und Engels schrieben im Kommunistischen Manifest, dass alles Stehende und Ständische verdampfe - oder, in der zweifellos poetischeren englischen Übersetzung, >everything that is solid melts into air $<$. Die Phrase ist seitdem zu einem Schlüsselzitat in der Beschreibung von Modernisierung geworden. Meinte es bei Marx und Engels die Auflösung der traditionellen Gesellschaft, lässt es sich natürlich ebenso auf die Modernisierung der Ersten Moderne anwenden. Allein, es scheint als ob der Bereich der Wirtschaft - und hier vor allem die Finanzmärkte - eine Ausnahme darstellt. >Marktkräfte< stehen als Fels in der Brandung der zweiten Moderne. Was zunächst als Anomalie erscheint ist vielleicht kein Zufall. Die Selbstreferentialität von Märkten macht es ihnen unmöglich, die eigene Reflexivität zu reflektieren. Während um sie herum alte Gewissheiten wegbrechen, stellen sie ein Reservoir an Ersatz-Eindeutigkeiten und Orientierung dar, aus dem heutige Gesellschaften immer öfter schöpfen. Aus soziologischer Perspektive ist es unwahrscheinlich, dass Gesellschaften in allen Lebensbereichen gleichzeitig Denkstrukturen aufgeben, die ihnen bis dato Halt gegeben haben. Wenn das doch geschieht, so wie beim Anbruch der ersten Moderne, müssen alte Reflexionsmuster durch neue ersetzt werden, die ein ähnliches Maß an Eindeutigkeiten und Orientierung bereithalten. Problematisch an der Zweiten Moderne ist nun, dass durch Anerkennung der Selbstreferentialität sozialen Handels die Eindeutigkeiten verschwinden. (Finanz-)Märkte werden dann zum blinden Fleck einer zweiten Moderne. Sie werden zur notwendigen Voraussetzung der Selbstreflexion in anderen Gesellschaftsbereichen, denn das Ökonomische stellt den (scheinbar) festen Boden bereit, auf dem wir stehen.

\section{Literaturhinweise}

Armijo, Leslie Elliott (2001): The Political Geography of World Financial Reform: Who Wants What and Why?, in: Global Governance 7, S. 379-396.

Baecker, Dirk (1991): Womit handeln Banken?, Frankfurt am Main.

--- (1999): Die Preisbildung an der Börse, in: Soziale Systeme 5, S. 287-312.

Beck, Ulrich (1986): Risikogesellschaft, Frankfurt am Main.

Beck, Ulrich / Bonß, Wolfgang / Lau, Christoph (2001): Theorie reflexiver Modernisierung - Fragestellungen, Hypothesen, Forschungsprogramme, in: Ulrich Beck / Wolfgang Bonß (Hrsg.), Die Modernisierung der Moderne, Frankfurt, S. 11-59.

--- (2003): The Theory of Reflexive Modernization: Problematic, Hypotheses and Research Programme, in: Theory, Culture \& Society 20, S. 1-33.

Beck, Ulrich / Giddens, Anthony / Lash, Scott (Hrsg.) (1994): Reflexive Modernisation. Politics, Tradition and Aesthetics in the Modern Social Order, Cambridge.

Beck, Ulrich / Holzer, Boris (2004): Reflexivität und Reflexion, in: Ulrich Beck / Christoph Lau (Hrsg.), Entgrenzung und Entscheidung, Frankfurt, S. 165-192.

11) In der Wochenendausgabe der Financial Times zum Beispiel erfreut sich die Rubrik >Adam Smith beantwortet Fragen des täglichen Lebens großer Beliebtheit. Leser fragen hier um Rat zu Themen die von Kindererziehung bis zur Partnerwahl reichen. Ungeachtet seines unterhaltsamen Charakters ist dieses Feature symptomatisch für einen tieferliegenden gesellschaftlichen Trend. 
Camdessus, Michel (1999): Reflections on the Crisis in Asia, Rede vor dem Extraordinary Ministerial Meeting der Gruppe der 24, Caracas, Venezuela, 7. Februar 1998.

Cerny, Philip (1990): The Changing Architecture of Politics: Structure, Agency, and the Future of the State, London.

Corsetti, Giancarlo / Pesenti, Paolo / Roubini, Nouriel (1998): What caused the Asian Currency and Financial Crisis?, Cambridge.

Crotty, Jim / Dymski, Gary (1998): Can the Global Neoliberal Regime survive Victory in Asia?, Dagenham.

Council on Foreign Relations Independent Task Force (1999): Safeguarding Prosperity in a Global Financial System: The Future International Financial Architecture, Council on Foreign Relations Independent Task Force, Washington DC.

de Goede, Marieke (2001): Discourses of Scientific Finance and the Failure of Long-Term Capital Management, in: New Political Economy 6, S. 149-170.

Dunbar, Nicholas (2000): Inventing Money: the story of long-term capital management and the legends behind it, Chichester.

Eichengreen, Barry (1999): Towards a New International Financial Architecture, Washington DC: Institute for International Economics.

Engelen, Ewald (1995): De Mythe van de Markt: Waarheid en Leugen in de Economie, Amsterdam.

Feldstein, Martin (1998): Refocusing the IMF, in: Foreign Affairs 77, S. 20-33.

- (1999): Self-Protection for Emerging Market Economies, Cambridge.

Financial Times (2004): Accounting chief warns of uproar over next stage of harmonisation, November 10, 2004.

- (2004): Agencies win early victory in review of regulation, December 8, 2004.

- (2004): Business chiefs attack Sarbanes-Oxley, 19. Oktober 2004.

- (2004): Scandal adds to rating agency woes, 12. Januar 2004.

Fligstein, Neil (2001): The Architecture of Markets: An Economic Sociology of Twenty-First-Century Capitalist Societies, Princeton.

Frankel, Jeffrey (1998): The Asian Model, the Miracle, the Crisis and the Fund, Rede vor der US International Trade Commission, 16.April 1998, Washington D.C.

G7 Finance Ministers (1999): Strengthening the International Financial Architecture. Report of the G7 Finance Ministers to the Köln Economic Summit, 18-20 June, 1999, Cologne.

Galbraith, John Kenneth (1975): Money. Whence it came, where it went, Harmondsworth.

Généreux, Jacques (2005): Les Vraies Lois de l'Èconomie, Paris.

Germain, Randall (2001): Global Financial Governance and the Problem of Inclusion, in: Global Governance 7, S. 411-426.

Granovetter, Mark (1985): Economic Action and Social Structure: The Problem of Embeddedness, in: American Journal of Sociology 91, S. 481-510.

Hall, Peter (1993): Policy paradigms, social learning and the state: the case of economic policy making in Britain, in: Comparative Politics 25, S. 275-296.

Handelsblatt (2004): S\&P stellt sich auf strengere Regeln ein, 29. Januar 2004.

IWF (1998a): World Economic Outlook October 1998, Washington D.C.: IMF.

- (1998b): The Asian Crisis: Causes and Cures, in: Finance and Development 35. http://www.imf.org/ external/pubs/ft/fandd/1998/06/imfstaff.htm (4. Juni 2003)

- (1999): The IMF's Response to the Crisis, veröffentlicht am 17. Januar 1999, Washington DC.

James, Harold (2001): The End of Globalization: Lessons from the Great Depression, Cambridge.

Kindleberger, Charles (1978): Manias, panics and crashes: a history of financial crises, London. 
Knowles, James / Pernia, Ernesto / Racelis, Mary (1999): Social Consequences of the Financial Crisis in Asia, Manila.

Krugman, Paul (2000): The Return of Depression Economics, London.

Latour, Bruno (1993): We have never been modern, Cambridge.

Mandelbrot, Benoit / Hudson, Richard (2004): The Misbehavior of Markets, New York.

Mitchell, Timothy (2002): Rule of Experts: Egypt, Techno-Politics, Modernity, Berkeley.

Nitzan, Jonathan (1998): Differential accumulation: towards a new political economy of capital, in: Review of International Political Economy 5, S. 169-216.

Partnoy, Frank (2002): Infectious greed: how deceit and risk corrupted the financial markets, New York.

Pauly, Louis (2002): Global finance, political authority, and the problem of legitimation, in: Rodney Bruce Hall / Thomas Biersteker (Hrsg.), The Emergence of Private Authority in Global Governance, Cambridge, S. 76-90.

Polanyi, Karl (2001 [1944]): The Great Transformation, Boston.

Rorty, Richard (1992): Kontingenz, Ironie und Solidarität, Frankfurt.

Sachs, Jeffrey / Radelet, Steven (1998): The Onset of the Asian Financial Crisis, Cambridge.

Shonfield, Andrew (1965): Modern Capitalism. The Changing Balance of Public and Private Power, Oxford.

Soederberg, Susanne (2002): On the contradictions of the New International Financial Architecture: another procrustean bed for emerging markets?, in: Third World Quarterly 23, S. 607-620.

Soros, George (1998): Die Krise des globalen Kapitalismus, Frankfurt.

Strange, Susan (1986): Casino Capitalism, Oxford.

- (1998): Mad Money, Manchester.

Stringham, Edward (2003): The extralegal development of securities trading in seventeenth-century Amsterdam, in: The Quarterly Review of Economics 43, S. 321-344.

Strulik, Thorsten (2000): Funktionen und Folgen privater Rating-Agenturen im Kontext der Regulierung globaler Finanzmärkte, in: Soziale Welt 51, S. 443-462.

Swedberg, Richard (2003): Principles of Economic Sociology, Princeton.

True, James / Jones, Bryan / Baumgartner, Frank (1999): Punctuated Equilibrium Theory: Explaining Stability and Change in American Policy Making, in: Paul Sabatier (Hrsg.), Theories of the Policy Process, Boulder, S. 97-115.

Underhill, Geoffrey (2001): The public good versus private interests and the global financial and monetary system, in: Daniel Drache (Hrsg.), Market or Public Domain? Global governance and the asymmetry of power, London, S. 274-295.

Zysman, John (1983): Governments, markets, and growth, Ithaca.

Daniel Mügge, Amsterdam School for Social Science Research, Universiteit van Amsterdam, Kloveniersburgwal 48, NL-1012 CX Amsterdam, Niederlande. d.k.muegge@uva.nl 